This is the final peer-reviewed accepted manuscript of:

Giovanni Cupini, Flavia Giannetti, Raffaella Giova, Antonia Passarelli di Napoli, Higher integrability for minimizers of asymptotically convex integrals with discontinuous coefficients, Nonlinear Analysis: Theory, Methods \& Applications, Volume 154, 2017, Pages 7-24, ISSN 0362-546X.

The final published version is available online at: https://doi.org/10.1016/j.na.2016.02.017

Rights / License:

The terms and conditions for the reuse of this version of the manuscript are specified in the publishing policy. For all terms of use and more information see the publisher's website.

This item was downloaded from IRIS Università di Bologna (https://cris.unibo.it/)

When citing, please refer to the published version. 


\title{
HIGHER INTEGRABILITY FOR MINIMIZERS OF ASYMPTOTICALLY CONVEX INTEGRALS WITH DISCONTINUOUS COEFFICIENTS
}

\author{
GIOVANNI CUPINI - FLAVIA GIANNETTI - RAFFAELLA GIOVA - ANTONIA PASSARELLI DI NAPOLI
}

This paper is dedicated to Nicola Fusco, who directed us to the theory of regularity in the Calculus of Variations, on the occasion of his 60th birthday.

\begin{abstract}
We study the local regularity of vectorial minimizers of integral functionals with standard $p$ growth. We assume that the non-homogeneous densities are uniformly convex and have a radial structure, with respect to the gradient variable, only at infinity. Under a $W^{1, n}$-Sobolev dependence on the spatial variable of the integrand, $n$ being the space dimension, we show that the minimizers have the gradient locally in $L^{q}$ for every $q>p$. As a consequence, they are locally $\alpha$-Hölder continuous for every $\alpha<1$.
\end{abstract}

\section{INTRODUCTION}

In this paper we study the regularity of vectorial local minimizers of functionals with irregular integrands in the $x$-variable and only asymptotically convex with respect to the gradient variable. In order to state our result precisely, we introduce right now our hypotheses. We will consider

$$
\mathcal{F}(u ; \Omega):=\int_{\Omega} f(x, D u) d x
$$

where $\Omega \subset \mathbb{R}^{n}, n>2$, is a bounded open set, $u: \Omega \rightarrow \mathbb{R}^{N}, N>1$, is a Sobolev map and $f$ : $\Omega \times \mathbb{R}^{n N} \rightarrow[0,+\infty)$ is a Carathéodory function convex with respect to the second variable.

As it is well known since the famous example by De Giorgi [13] (see also [39], [40], [45]), in order to avoid the irregularity phenomena peculiar of the vectorial minimizers, the dependence of the energy density on the modulus of the gradient variable is necessary. We shall assume it only at infinity, i.e., for large values of the gradient variable $\xi$. Precisely:

(A1) there exist $\tilde{R}>0$ and a function $\tilde{f}: \Omega \times[\tilde{R},+\infty) \rightarrow[0,+\infty)$ such that

$$
f(x, \xi)=\tilde{f}(x,|\xi|) \text {, }
$$

for a.e. $x \in \Omega$ and every $\xi \in \mathbb{R}^{n N} \backslash B_{\tilde{R}}(0)$.

The integrand $f$ will satisfy the so-called $p$-growth condition, that is

(A2) there exist an exponent $p>1$ and constants $c_{1}, c_{2}, L>0$ such that

$$
c_{1}|\xi|^{p}-c_{2} \leq f(x, \xi) \leq L(1+|\xi|)^{p},
$$

for a.e. $x \in \Omega$ and $\xi \in \mathbb{R}^{n N}$.

2000 Mathematics Subject Classification. Primary: 49N60; 49N99. Secondary: 35 J47.

Key words and phrases. Regularity, asymptotically convex, minimizer, $p$-growth.

Acknowledgement: The first and the last authors have been supported by MIUR through the Project PRIN (2012) "Calcolo delle Variazioni". The third author has been partially supported by Project Legge 5/2007 Regione Campania "Spazi pesati ed applicazioni al calcolo delle variazioni". All the authors have been supported by the Gruppo Nazionale per l'Analisi Matematica, la Probabilità e le loro Applicazioni (GNAMPA) of the Istituto Nazionale di Alta Matematica (INdAM). 
The usual p-uniform convexity will be assumed only at infinity. More precisely, we shall suppose that $\xi \rightarrow f(x, \xi) \in C^{2}\left(\mathbb{R}^{n N} \backslash B_{\tilde{R}}(0)\right)$ and

(A3) there exists $\nu>0$ such that

$$
\left\langle D_{\xi \xi} f(x, \xi) \lambda, \lambda\right\rangle \geq \nu(1+|\xi|)^{p-2}|\lambda|^{2},
$$

for a.e. $x \in \Omega$, for every $\xi \in \mathbb{R}^{n N} \backslash B_{\tilde{R}}(0)$ and for every $\lambda \in \mathbb{R}^{n N}$.

Note that, since $f$ is $C^{2}$ with respect to the gradient variable outside the ball $B_{\tilde{R}}(0)$, the assumption in (A3) is equivalent to the $C^{2}$ - asymptotic convexity introduced in [5].

Also the bound on the second order derivatives in the gradient variable will be required only at infinity. Indeed, we shall assume that

(A4) there exists $L_{1}>0$ such that

$$
\left|D_{\xi \xi} f(x, \xi)\right| \leq L_{1}(1+|\xi|)^{p-2},
$$

for a.e. $x \in \Omega$ and every $\xi \in \mathbb{R}^{n N} \backslash B_{\tilde{R}}(0)$.

We now introduce the main property of our energy density. As already mentioned, we will not ask a regular dependence of $f$ on the $x$-variable. Indeed, the function $x \rightarrow D_{\xi} f(x, \xi)$ will be required to be weakly differentiable for every $\xi \in \mathbb{R}^{n N} \backslash B_{\tilde{R}}(0)$ and it will be assumed that

(A5) there exists a non-negative function $k \in L_{\text {loc }}^{n}(\Omega)$ such that

$$
\left|D_{\xi x} f(x, \xi)\right| \leq k(x)(1+|\xi|)^{p-1},
$$

for a.e. $x \in \Omega$ and for every $\xi \in \mathbb{R}^{n N} \backslash B_{\tilde{R}}(0)$.

If we take into account that many properties are requested only at infinity, a model functional in our setting is

$$
\mathcal{F}(u ; \Omega):=\int_{\Omega} a(x)|D u|^{p} d x, \quad p>1
$$

with $a \in W_{\text {loc }}^{1, n}(\Omega) \cap L^{\infty}(\Omega), a \geq 1$.

Let us now briefly discuss our assumptions.

We begin noting that the counterpart of the uniform $p$-convexity at infinity, in case of non-smooth functions $f$ is that there exists $\nu>0$ such that

$$
\frac{1}{2}\left[f\left(x, \xi_{1}\right)+f\left(x, \xi_{2}\right)\right] \geq f\left(x, \frac{\xi_{1}+\xi_{2}}{2}\right)+\nu\left(1+\left|\xi_{1}\right|^{2}+\left|\xi_{2}\right|^{2}\right)^{\frac{p-2}{2}}\left|\xi_{1}-\xi_{2}\right|^{2},
$$

for a.e. $x \in \Omega$ and for every $\xi_{1}, \xi_{2} \in \mathbb{R}^{n N} \backslash B_{\tilde{R}}(0)$ endpoints of a segment contained in the complement of $B_{\tilde{R}}(0)$. This property was introduced by Fonseca, Fusco and Marcellini in [18] in order to study the existence of real valued minimizers of some non convex variational problems. Already in such a paper, the condition (1.3) has been revealed sufficient for the Lipschitz regularity of local minimizers. Since then, the regularity properties of local minimizers of $\mathcal{F}(u ; \Omega)$ when the integrand $f$ is $p$-uniformly convex at infinity with respect to the the gradient variable have been widely investigated assuming a dependence on the $x$ variable through a smooth function (see [4], [9], [10], [17], [20], [21], [22], [23], [24], [44]).

On the other hand, many regularity results are available when the integrand $f$ is uniformly convex with respect to the last variable but discontinuous with respect to $x$. In particular, when the dependence on $x$ is given through a coefficient belonging to a suitable Sobolev class, the higher differentiability as well as the partial Hölder continuity of the gradient of the local minimizers have been obtained in [28], [32], [41], [42], [43]. More recently, the regularity of the solutions of some parabolic systems with Sobolev coefficients has been faced in [30]. We refer to [27], [29] for the case of functionals with variable exponents growth condition. 
The aim of this paper is to show that the Sobolev dependence on the $x$-variable expressed by the assumption (A5) is sufficient to prove that, under a $p$-convexity condition only at infinity, the local minimizers $u$ of $\mathcal{F}$ have the gradient locally in $L^{q}$ for every $q>p$ and, therefore, that they are locally Hölder continuous for every exponent $0<\alpha<1$.

More precisely, taking into account that, without loss of generality, we can assume $\tilde{R}=1$, our main result is the following.

Theorem 1.1. Let $f: \Omega \times \mathbb{R}^{n N} \rightarrow[0,+\infty)$ be a Carathéodory function such that $f=f(x, \xi)$ is convex and $C^{2}$ with respect to the last variable and satisfies the assumptions (A1)-(A5), with $p>1$. Let $u \in W_{\mathrm{loc}}^{1, p}\left(\Omega, \mathbb{R}^{N}\right)$ be a local minimizer of (1.1). Then $u$ is locally $\alpha$-Hölder continuous for all $\alpha \in(0,1)$. Moreover, for all $q>p$ and for all $B_{\rho}\left(x_{0}\right) \subset B_{R}\left(x_{0}\right) \Subset \Omega$ we have that

$$
\left[\int_{B_{\rho}\left(x_{0}\right)}|D u|^{q} d x\right]^{\frac{1}{q}} \leq C\left[\int_{B_{R}\left(x_{0}\right)}(1+f(x, D u)) d x\right]^{\frac{1}{p}},
$$

where $C=C\left(n, N, p, q, L, L_{1}, \nu, c_{1}, c_{2}, \rho, R\right)$.

We remark that Hölder continuity results for any exponent $\alpha$ strictly less than 1 , are not uncommon when the integrands depend on $x$. We refer to [8] and [42] for examples of not locally Lipschitz continuous minimizers, but Hölder continuous for every exponent.

It is worth pointing out that we were inspired by a regularity result proved in [14] for functionals satisfying the non-standard $(p, q)$-growth conditions. We recall that the theory of regularity of minimizers in this framework of non-standard growths was started by Marcellini, see [36], [37], see also AcerbiFusco [2], Boccardo-Marcellini-Sbordone [3], Fusco-Sbordone [25], [26], and later widely investigated by many authors and in different settings of applicability. For more details and references on this subject we refer to [38].

Besides the $(p, q)$-growth condition, Eleuteri, Marcellini and Mascolo in [14] deal with an integrand $f$ satisfying (1.2) for all $\xi \in \mathbb{R}^{n N}$, but satisfying the assumption (A5) with a function $k$ belonging to $L^{r}$, with $r>n$. They proved that the local minimizers of the integrals are locally Lipschitz continuous if $\frac{q}{p}<1+\frac{1}{n}-\frac{1}{r}$ (note that this assumption on the gap $\frac{q}{p}$ is sharp as shown in [16] and [19]). See also the papers by Colombo and Mingione [6], [7] for related results. We also cite the paper [34] by Kristensen and Mingione, see also Kuusi and Mingione [35], for other interesting regularity results for vector valued minimizers under weak properties in the $x$-dependence of the energy density.

Even though the proof of Theorem 1.1 relies on analogous arguments to those in [14], it seems that under the weaker assumption $k \in L_{\mathrm{loc}}^{n}(\Omega)$ the local Lipschitz continuity of the minimizers cannot be achieved even if $p=q$.

As in [14], we shall combine a suitable a priori estimate for the gradient of the local minimizers with an approximation procedure. As a common feature, we shall establish the a priori estimate for smooth minimizers and then we will show that such a priori estimate is preserved in passing to the limit.

Note that, in order to face new difficulties arising from our weaker assumptions on the function $k$, to obtain the apriori estimate we shall use a double iteration procedure that will allow us to reabsorb certain integrals involving the gradient with a critical integrability exponent. Moreover, in our approximation procedure will have to take into account the lack of convexity of the integrand for small values of the gradient.

Remark 1.2. When $f$ satisfies the assumptions of Theorem 1.1, but is not convex in the last variable, the local minimizers of $\mathcal{F}$ still satisfies the thesis of the previous theorem, provided

$$
\operatorname{sc}^{-} \mathcal{F}(w ; \Omega)=\int_{\Omega} f^{* *}(x, D w) d x
$$


where $\mathrm{sc}^{-} \mathcal{F}$ is the relaxed functional of $\mathcal{F}$ and $\xi \mapsto f^{* *}(x, \xi)$ is the convex envelope of $f(x, \cdot)$. In fact, $f^{* *}$ fulfills (A1), (A2), (A3), (A4) with suitable constants $\nu$ and $R$. Moreover, $f=f^{* *}$ in $\Omega \times B_{\bar{R}}$ for some $\bar{R} \geq \tilde{R}$ (see e.g. Theorem 2.5 (iv) in [9]) therefore (A5) holds for $f^{* *}$, with $\bar{R}$ replacing $\tilde{R}$. Since $\mathrm{sc}^{-} \mathcal{F}$ is the relaxed functional, if $u$ is a local minimizer of $\mathcal{F}$, then $u$ is a local minimizer of $\mathrm{sc}^{-} \mathcal{F}$, too. As said, the integrand $f^{* *}$ satisfies the assumptions of Theorem 1.1; thus Theorem 1.1 applies and we get that the gradient of $u$ is in $L_{\mathrm{loc}}^{q}(\Omega)$ for every finite $q>p$.

The plan of the paper is the following: in Section 2 we fix the notations and collect preliminary results; in Section 3 we establish the a priori estimate and in Section 4 we give the proof of the main result.

\section{PRELIMINARIES}

In this section we recall some standard definitions and collect several lemmas that we shall use to establish our main result.

First of all, we recall that $u \in W_{\text {loc }}^{1, p}\left(\Omega, \mathbb{R}^{N}\right)$ is a local minimizer of $\mathcal{F}$ in (1.1) if

$$
\mathcal{F}(u ; \operatorname{supp} \varphi) \leq \mathcal{F}(u+\varphi ; \operatorname{supp} \varphi)
$$

for any $\varphi \in W^{1, p}\left(\Omega, \mathbb{R}^{N}\right)$ with $\operatorname{spt} \varphi \Subset \Omega$.

We will follow the usual convention and denote by $C$ a general constant that may vary on different occasions, even within the same line of estimates. Relevant dependencies on parameters and special constants will be suitably emphasized using parentheses or subscripts. All the norms we use on $\mathbb{R}^{n}, \mathbb{R}^{N}$ and $\mathbb{R}^{n N}$ will be the standard Euclidean ones and denoted by $|\cdot|$ in all cases. In particular, for matrices $\xi, \eta \in \mathbb{R}^{n N}$ we write $\langle\xi, \eta\rangle:=\operatorname{trace}\left(\xi^{T} \eta\right)$ for the usual inner product of $\xi$ and $\eta$, and $|\xi|:=\langle\xi, \xi\rangle^{\frac{1}{2}}$ for the corresponding euclidean norm.

In what follows, $B_{r}^{n}(x)$ will denote the ball in $\mathbb{R}^{n}$ centered at $x$ of radius $r$. Analogously, $B_{r}^{n N}(\xi)$ is a ball in $\mathbb{R}^{n N}$. If no confusion may arise we shall omit the superscripts related to the space dimension and the dependence on the center.

The following lemma finds an important application in the so called hole-filling method. Its proof can be found for example in [33, Lemma 6.1] .

Lemma 2.1. Let $h:\left[r, R_{0}\right] \rightarrow \mathbb{R}$ be a non-negative bounded function and $0<\vartheta<1, A, B \geq 0$ and $\beta>0$. Assume that

for all $r \leq s<t \leq R_{0}$. Then

$$
h(s) \leq \vartheta h(t)+\frac{A}{(t-s)^{\beta}}+B,
$$

$$
h(r) \leq \frac{c A}{\left(R_{0}-r\right)^{\beta}}+c B
$$

where $c=c(\vartheta, \beta)>0$.

We shall need the following

Lemma 2.2. Let $u \in W_{\mathrm{loc}}^{1, p}\left(\Omega, \mathbb{R}^{N}\right)$ be a local minimizer of

$$
\mathcal{F}(w ; \Omega)=\int_{\Omega} f(x, D w(x)) d x,
$$

where $f: \Omega \times \mathbb{R}^{n N} \rightarrow[0,+\infty)$ is a Carathéodory function, convex with respect to the last variable, satisfying (A2) and (A3). Suppose that there exist $B_{r}\left(x_{0}\right) \Subset \Omega$ and $v \in u+W_{0}^{1,1}\left(B_{r}\left(x_{0}\right), \mathbb{R}^{N}\right)$ such that $\mathcal{F}\left(u ; B_{r}\left(x_{0}\right)\right)=\mathcal{F}\left(v ; B_{r}\left(x_{0}\right)\right)$. Then there exists $R_{0}(p, \nu, \tilde{R}, L)>\tilde{R}$ such that the Lebesgue measure of the set

$$
\left\{x \in B_{r}\left(x_{0}\right):|D u(x)+D v(x)|>2 R_{0} \text { and }|D u(x)-D v(x)|>0\right\}
$$


is zero.

For the proof we refer to [9]. We conclude recalling a regularity result for minimizers of functionals whose integrands are smooth with respect to the $x$-variable (see e.g. [31] for the case $p \geq 2$ and [1] for the case $1<p<2$; see also [15] and, in particular, Theorem 1.1 in [9] as far as the Lipschitz continuity of the local minimizers is concerned).

Lemma 2.3. Let $u \in W_{\text {loc }}^{1, p}\left(\Omega, \mathbb{R}^{N}\right)$ be a local minimizer of the functional at (1.1), with $f: \Omega \times \mathbb{R}^{n N} \rightarrow$ $[0,+\infty), f \in C^{2}\left(\Omega \times \mathbb{R}^{n N}\right)$. Assume that there exists $p>1$ such that for every $x \in \Omega$ and every $\xi, \lambda \in \mathbb{R}^{n N}$,

$$
\begin{gathered}
c_{1}|\xi|^{p}-c_{2} \leq f(x, \xi) \leq L(1+|\xi|)^{p}, \\
\nu(1+|\xi|)^{p-2}|\lambda|^{2} \leq\left\langle D_{\xi \xi} f(x, \xi) \lambda, \lambda\right\rangle, \\
\left|D_{\xi \xi} f(x, \xi)\right| \leq L_{1}(1+|\xi|)^{p-2}, \\
\left|D_{\xi x} f(x, \xi)\right| \leq K(1+|\xi|)^{p-1},
\end{gathered}
$$

with positive constants $c_{1}, c_{2}, L, L_{1}, \nu, K$.

Then $u \in W_{\mathrm{loc}}^{2, a}(\Omega)$, with $a=\min \{2, p\}$ and $\left(1+|D u|^{2}\right)^{\frac{p-2}{2}}\left|D^{2} u\right|^{2} \in L_{\mathrm{loc}}^{1}(\Omega)$. If, moreover, (A1) holds, then $u \in W_{\mathrm{loc}}^{1, \infty}(\Omega)$ and $u \in W_{\mathrm{loc}}^{2,2}(\Omega)$.

\section{THE A PRIORI ESTIMATE}

The following a priori estimate is the key tool for our main result. Its proof follows the one of [14, Proposition 3.1], but it is drastically different in the second step were we have to take into account the weaker assumption on the function $k(x)$ appearing in (A5).

Theorem 3.1. Let $u \in W_{\text {loc }}^{1, p}\left(\Omega, \mathbb{R}^{N}\right)$ be a local minimizer of the functional $\mathcal{F}$ at (1.1) with the integrand $f$ satisfying the assumptions (A1)-(A5). If $u \in W_{\mathrm{loc}}^{2, \min \{2, p\}}\left(\Omega ; \mathbb{R}^{N}\right) \cap W_{\mathrm{loc}}^{1, \infty}\left(\Omega ; \mathbb{R}^{N}\right)$ and $\left(1+|D u|^{2}\right)^{\frac{p-2}{2}}\left|D^{2} u\right|^{2} \in L_{\mathrm{loc}}^{1}(\Omega)$, then, for every exponent $q \geq p$ and for all $x_{0} \in \Omega$, there exist a radius $R_{q}=R_{q}\left(n, N, p, q, \nu, k, L, L_{1}, x_{0}\right)$ and a ball $B_{R_{q}}\left(x_{0}\right) \Subset \Omega$ such that the following estimate

$$
\left[\int_{B_{\rho}\left(x_{0}\right)}|D u|^{q} d x\right]^{\frac{1}{q}} \leq C\left[\int_{B_{R}\left(x_{0}\right)}(1+f(x, D u)) d x\right]^{\frac{1}{p}},
$$

holds for every $\rho$ and $R$, with $0<\rho<R<R_{q}$, and with a positive constant $C=$ $C\left(n, N, p, q, L, L_{1}, \nu, c_{1}, c_{2}, \rho, R\right)$.

Proof. We shall divide the proof into three steps.

Step 1. Our first aim is to prove that, for every $\gamma \geq 0$ and for every cut off function $\eta \in C_{0}^{\infty}(\Omega)$, the following integral estimate holds

$$
\begin{aligned}
& \left.\int_{\Omega} \eta^{2}\left(1+(|D u|-1)_{+}\right)^{\gamma-2}(|D u|-1)_{+}\right)^{2}|D u|^{p-2}\left|D^{2} u\right|^{2} d x \\
\leq & C(\gamma+1)^{2} \int_{\Omega} \eta^{2} k^{2}(x)\left(1+(|D u|-1)_{+}\right)^{\gamma+p} d x \\
+ & C \int_{\Omega}|D \eta|^{2}\left(1+(|D u|-1)_{+}\right)^{\gamma+p} d x,
\end{aligned}
$$

with $C=C\left(n, N, L, L_{1}, \nu, p\right)$. 
Since $u$ is a local minimizer of the functional $\mathcal{F}(u, \Omega)$, then $u$ satisfies the Euler's system

$$
\int_{\Omega} \sum_{i, \alpha} f_{\xi_{i}^{\alpha}}(x, D u) \varphi_{x_{i}}^{\alpha}(x) d x=0 \quad \forall \varphi \in C_{0}^{\infty}\left(\Omega ; \mathbb{R}^{N}\right),
$$

and, using the second variation, for every $s=1, \ldots, n$ it holds

$$
\int_{\Omega}\left\{\sum_{i, j, \alpha, \beta} f_{\xi_{i}^{\alpha} \xi_{j}^{\beta}}(x, D u) \varphi_{x_{i}}^{\alpha} u_{x_{s} x_{j}}^{\beta}+\sum_{i, \alpha} f_{\xi_{i}^{\alpha} x_{s}}(x, D u) \varphi_{x_{i}}^{\alpha}\right\} d x=0 \quad \forall \varphi \in C_{0}^{\infty}\left(\Omega ; \mathbb{R}^{N}\right) .
$$

Fix $s=1, \ldots, n$, an exponent $\gamma \geq 0$, a cut off function $\eta \in C_{0}^{\infty}(\Omega)$ and consider the function

$$
\varphi^{\alpha}:=\eta^{2} u_{x_{s}}^{\alpha}\left(1+(|D u|-1)_{+}\right)^{\gamma-2}\left((|D u|-1)_{+}\right)^{2} \quad \alpha=1, \ldots, N .
$$

Therefore, denoting

$$
\Phi(t):=(1+t)^{\gamma-2} t^{2}
$$

we have

$$
\begin{aligned}
\varphi_{x_{i}}^{\alpha} & =2 \eta \eta_{x_{i}} u_{x_{s}}^{\alpha} \Phi\left((|D u|-1)_{+}\right) \\
& +\eta^{2} u_{x_{s} x_{i}}^{\alpha} \Phi\left((|D u|-1)_{+}\right)+\eta^{2} u_{x_{s}}^{\alpha} \Phi^{\prime}\left((|D u|-1)_{+}\right)\left[(|D u|-1)_{+}\right]_{x_{i}} .
\end{aligned}
$$

Thanks to our assumptions on the minimizer $u$, through a standard density argument, we can use $\varphi$ as test function in the equation (3.2), thus getting

$$
\begin{aligned}
0 & =\int_{\Omega} 2 \eta \Phi\left((|D u|-1)_{+}\right) \sum_{i, j, s, \alpha, \beta} f_{\xi_{i}^{\alpha} \xi_{j}^{\beta}}(x, D u) \eta_{x_{i}} u_{x_{s}}^{\alpha} u_{x_{s} x_{j}}^{\beta} d x \\
& +\int_{\Omega} \eta^{2} \Phi\left((|D u|-1)_{+}\right) \sum_{i, j, s, \alpha, \beta} f_{\xi_{i}^{\alpha} \xi_{j}^{\beta}}(x, D u) u_{x_{s} x_{i}}^{\alpha} u_{x_{s} x_{j}}^{\beta} d x \\
& +\int_{\Omega} \eta^{2} \Phi^{\prime}\left((|D u|-1)_{+}\right) \sum_{i, j, s, \alpha, \beta} f_{\xi_{i}^{\alpha} \xi_{j}^{\beta}}(x, D u) u_{x_{s}}^{\alpha} u_{x_{s} x_{j}}^{\beta}\left[(|D u|-1)_{+}\right]_{x_{i}} d x \\
& +\int_{\Omega} 2 \eta \Phi\left((|D u|-1)_{+}\right) \sum_{i, s, \alpha} f_{\xi_{i}^{\alpha} x_{s}}(x, D u) \eta_{x_{i}} u_{x_{s}}^{\alpha} d x \\
& +\int_{\Omega} \eta^{2} \Phi\left((|D u|-1)_{+}\right) \sum_{i, s, \alpha} f_{\xi_{i}^{\alpha} x_{s}}(x, D u) u_{x_{s} x_{i}}^{\alpha} d x \\
& +\int_{\Omega} \eta^{2} \Phi^{\prime}\left((|D u|-1)_{+}\right) \sum_{i, s, \alpha} f_{\xi_{i}^{\alpha} x_{s}}(x, D u) u_{x_{s}}^{\alpha}\left[(|D u|-1)_{+}\right]_{x_{i}} d x \\
& =: I_{1}+I_{2}+I_{3}+I_{4}+I_{5}+I_{6} .
\end{aligned}
$$

Let us now estimate the integrals $I_{j}, j=1, \ldots, 6$.

ESTIMATE OF $I_{1}$

By (A3) and (A4), we can estimate the integral $I_{1}$ by the use of Cauchy-Schwartz and Young's inequalities as follows

$$
\left|I_{1}\right| \leq 2 \int_{\Omega} \Phi\left((|D u|-1)_{+}\right)\left\{\sum_{i, j, s, \alpha, \beta} f_{\xi_{i}^{\alpha} \xi_{j}^{\beta}}(x, D u) \eta_{x_{i}} u_{x_{s}}^{\alpha} \eta_{x_{j}} u_{x_{s}}^{\beta}\right\}^{\frac{1}{2}}\left\{\eta^{2} \sum_{i, j, s, \alpha, \beta} f_{\xi_{i}^{\alpha} \xi_{j}^{\beta}}(x, D u) u_{x_{s} x_{i}}^{\alpha} u_{x_{s} x_{j}}^{\beta}\right\}^{\frac{1}{2}}
$$




$$
\begin{aligned}
& \leq C\left(\varepsilon, L_{1}\right) \int_{\Omega}|D \eta|^{2} \Phi\left((|D u|-1)_{+}\right)|D u|^{p} d x \\
& +\varepsilon \int_{\Omega} \eta^{2} \Phi\left((|D u|-1)_{+}\right) \sum_{i, j, s, \alpha, \beta} f_{\xi_{i}^{\alpha} \xi_{j}^{\beta}}(x, D u) u_{x_{s} x_{i}}^{\alpha} u_{x_{s} x_{j}}^{\beta} d x,
\end{aligned}
$$

where $\varepsilon>0$ will be chosen later.

ESTIMATE OF $I_{3}$

\section{Since}

$$
f_{\xi_{i}^{\alpha} \xi_{j}^{\beta}}(x, \xi)=\left(\frac{\tilde{f}_{t t}(x,|\xi|)}{|\xi|^{2}}-\frac{\tilde{f}_{t}(x,|\xi|)}{|\xi|^{3}}\right) \xi_{i}^{\alpha} \xi_{j}^{\beta}+\frac{\tilde{f}_{t}(x,|\xi|)}{|\xi|} \delta_{\xi_{i}^{\alpha}} \xi_{j}^{\beta}
$$

and

$$
\left[(|D u|-1)_{+}\right]_{x_{i}}=(|D u|)_{x_{i}}=\frac{1}{|D u|} \sum_{\alpha, s} u_{x_{i} x_{s}}^{\alpha} u_{x_{s}}^{\alpha} \quad \text { a.e. in }\{|D u| \geq 1\}
$$

then, for a.e. $x \in\{|D u| \geq 1\}$,

$$
\begin{aligned}
& \sum_{i, j, s, \alpha, \beta} f_{\xi_{i}^{\alpha} \xi_{j}^{\beta}}(x, D u) u_{x_{s}}^{\alpha} u_{x_{s} x_{j}}^{\beta}\left[(|D u|-1)_{+}\right]_{x_{i}} \\
= & \left(\frac{\tilde{f}_{t t}(x,|D u|)}{|D u|^{2}}-\frac{\tilde{f}_{t}(x,|D u|)}{|D u|^{3}}\right) \sum_{i, j, s, \alpha, \beta} u_{x_{s}}^{\alpha} u_{x_{s} x_{j}}^{\beta} u_{x_{i}}^{\alpha} u_{x_{j}}^{\beta}\left[(|D u|-1)_{+}\right]_{x_{i}} \\
+ & \frac{\tilde{f}_{t}(x,|D u|)}{|D u|} \sum_{i, s, \alpha} u_{x_{s}}^{\alpha} u_{x_{s} x_{i}}^{\alpha}\left[(|D u|-1)_{+}\right]_{x_{i}} \\
= & \left(\frac{\tilde{f}_{t t}(x,|D u|)}{|D u|}-\frac{\tilde{f}_{t}(x,|D u|)}{|D u|^{2}}\right) \sum_{i, s, \alpha} u_{x_{s}}^{\alpha}(|D u|)_{x_{s}} u_{x_{i}}^{\alpha}(|D u|)_{x_{i}} \\
+ & \frac{\tilde{f}_{t}(x,|D u|)}{|D u|} \sum_{i, s, \alpha} u_{x_{s}}^{\alpha} u_{x_{s} x_{i}}^{\alpha}(|D u|)_{x_{i}} \\
= & \left(\frac{\tilde{f}_{t t}(x,|D u|)}{|D u|}-\frac{\tilde{f}_{t}(x,|D u|)}{|D u|^{2}}\right) \sum_{\alpha}\left(\sum_{i} u_{x_{i}}^{\alpha}(|D u|)_{x_{i}}\right)^{2} \\
+ & \tilde{f}_{t}(x,|D u|)|D(|D u|)|^{2} .
\end{aligned}
$$

Thus,

$$
\begin{aligned}
I_{3}=\int_{\Omega} \eta^{2} \Phi^{\prime}\left((|D u|-1)_{+}\right) & \left\{\left(\frac{\tilde{f}_{t t}(x,|D u|)}{|D u|}-\frac{\tilde{f}_{t}(x,|D u|)}{|D u|^{2}}\right) \sum_{\alpha}\left(\sum_{i} u_{x_{i}}^{\alpha}(|D u|)_{x_{i}}\right)^{2}\right. \\
& \left.+\tilde{f}_{t}(x,|D u|)|D(|D u|)|^{2}\right\} d x
\end{aligned}
$$

Now, if we use the Cauchy-Schwartz inequality, we have

$$
\sum_{\alpha}\left(\sum_{i} u_{x_{i}}^{\alpha}(|D u|)_{x_{i}}\right)^{2} \leq|D u|^{2}|D(|D u|)|^{2}
$$

and observing that

$$
\Phi^{\prime}\left((|D u|-1)_{+}\right) \geq 0 \quad \text { and } \quad \tilde{f}_{t}(x,|D u|) \geq 0
$$


we conclude

$$
I_{3} \geq \int_{\Omega} \eta^{2} \Phi^{\prime}\left((|D u|-1)_{+}\right) \frac{\tilde{f}_{t t}(x,|D u|)}{|D u|} \sum_{\alpha}\left(\sum_{i} u_{x_{i}}^{\alpha}(|D u|)_{x_{i}}\right)^{2} d x \geq 0 .
$$

ESTIMATE OF $I_{4}$

By using the assumption (A5) we obtain

$$
\begin{aligned}
\left|I_{4}\right| & \leq 2^{p-1} \int_{\Omega} \eta \Phi\left((|D u|-1)_{+}\right) k(x)|D u|^{p-1} \sum_{i, s, \alpha}\left|\eta_{x_{i}} u_{x_{s}}^{\alpha}\right| d x \\
& \leq 2^{p-1} \int_{\Omega} \eta|D \eta| k(x) \Phi\left((|D u|-1)_{+}\right)|D u|^{p} d x .
\end{aligned}
$$

ESTIMATE OF $I_{5}$

Using the assumption (A5) and Young's inequality we have that

$$
\begin{aligned}
\left|I_{5}\right| & \leq 2^{p-1} \int_{\Omega} \eta^{2} \Phi\left((|D u|-1)_{+}\right) k(x)|D u|^{p-1}\left|D^{2} u\right| d x \\
& \leq \varepsilon \int_{\Omega} \eta^{2} \Phi\left((|D u|-1)_{+}\right)|D u|^{p-2}\left|D^{2} u\right|^{2} d x \\
& +C_{\varepsilon} \int_{\Omega} \eta^{2} \Phi\left((|D u|-1)_{+}\right) k^{2}(x)|D u|^{p} d x,
\end{aligned}
$$

where $\varepsilon \in(0,1)$ will be chosen later.

ESTIMATE OF $I_{6}$

Using the assumption (A5) and (3.5), we get

$$
\begin{aligned}
\left|I_{6}\right| & \leq 2^{p-1} \int_{\Omega} \eta^{2} \Phi^{\prime}\left((|D u|-1)_{+}\right) k(x)|D u|^{p-1}|D u|\left|D\left((|D u|-1)_{+}\right)\right| d x \\
& \leq C \int_{\Omega} \eta^{2} \Phi^{\prime}\left((|D u|-1)_{+}\right) k(x)|D u|^{p}\left|D^{2} u\right| d x
\end{aligned}
$$

Now, since

$$
\Phi^{\prime}(t)=(\gamma t+2) t(1+t)^{\gamma-3}
$$

it follows that

$$
t \Phi^{\prime}(t)=t(\gamma t+2) t(1+t)^{\gamma-3}=\frac{\gamma t+2}{1+t} \Phi(t) \leq 2(1+\gamma) \Phi(t) \quad \text { for all } t \geq 0 .
$$

Using the notation $c_{\gamma}:=2(1+\gamma)>0$, multiplying and dividing the integrand in the right hand side of (3.10) by $c_{\gamma}^{-1 / 2}\left(\delta+(|D u|-1)_{+}\right)^{1 / 2}$, with $0<\delta<1$ to be chosen later, we have

$$
\begin{aligned}
\left|I_{6}\right| & \leq \int_{\Omega} \eta^{2}\left\{\frac{1}{c_{\gamma}} \Phi^{\prime}\left((|D u|-1)_{+}\right)\left(\delta+(|D u|-1)_{+}\right)|D u|^{p-2}\left|D^{2} u\right|^{2}\right\}^{\frac{1}{2}} \\
& \times\left\{C^{2} c_{\gamma} \Phi^{\prime}\left((|D u|-1)_{+}\right) k^{2}(x)\left(\delta+(|D u|-1)_{+}\right)^{-1}|D u|^{p+2}\right\}^{\frac{1}{2}} d x \\
& \leq c_{\gamma} C_{\varepsilon} \int_{\Omega} \eta^{2} \Phi^{\prime}\left((|D u|-1)_{+}\right) k^{2}(x)\left(\delta+(|D u|-1)_{+}\right)^{-1}|D u|^{p+2} d x \\
& +\frac{\varepsilon}{c_{\gamma}} \int_{\Omega} \eta^{2} \Phi^{\prime}\left((|D u|-1)_{+}\right)\left(\delta+(|D u|-1)_{+}\right)|D u|^{p-2}\left|D^{2} u\right|^{2} d x
\end{aligned}
$$


where we used Young's inequality. In order to estimate the last integral in (3.12) we note that

$$
\delta+(|D u|-1)_{+} \leq 2(|D u|-1)_{+} \quad \text { a.e. in }\{|D u| \geq 2\},
$$

and, since $\Phi^{\prime}(t)$ is increasing, also that

$$
\Phi^{\prime}\left((|D u|-1)_{+}\right) \leq \Phi^{\prime}(2) \chi_{\{|D u| \geq 1\}} \quad \text { a.e. in }\{|D u| \leq 2\} .
$$

Therefore, by virtue of (3.11), we have

$$
\begin{aligned}
& \frac{\varepsilon}{c_{\gamma}} \int_{\Omega} \eta^{2} \Phi^{\prime}\left((|D u|-1)_{+}\right)\left(\delta+(|D u|-1)_{+}\right)|D u|^{p-2}\left|D^{2} u\right|^{2} d x \\
\leq & 2 \frac{\varepsilon}{c_{\gamma}} \int_{|D u| \geq 2} \eta^{2} \Phi^{\prime}\left((|D u|-1)_{+}\right)(|D u|-1)_{+}|D u|^{p-2}\left|D^{2} u\right|^{2} d x \\
+ & \frac{\varepsilon}{c_{\gamma}} \int_{|D u| \leq 2} \eta^{2} \Phi^{\prime}\left((|D u|-1)_{+}\right)(|D u|-1)_{+}|D u|^{p-2}\left|D^{2} u\right|^{2} d x \\
+ & \frac{\varepsilon \delta}{c_{\gamma}} \int_{|D u| \leq 2} \eta^{2} \Phi^{\prime}\left((|D u|-1)_{+}\right)|D u|^{p-2}\left|D^{2} u\right|^{2} d x \\
\leq & 2 \varepsilon \int_{\Omega} \eta^{2} \Phi\left((|D u|-1)_{+}\right)|D u|^{p-2}\left|D^{2} u\right|^{2} d x \\
+ & \frac{\varepsilon \delta}{c_{\gamma}} \int_{1 \leq|D u| \leq 2} \eta^{2} \Phi^{\prime}(2)|D u|^{p-2}\left|D^{2} u\right|^{2} d x \\
\leq & 2 \varepsilon \int_{\Omega} \eta^{2} \Phi\left((|D u|-1)_{+}\right)|D u|^{p-2}\left|D^{2} u\right|^{2} d x \\
+ & \frac{\varepsilon \delta}{2} \int_{|D u| \geq 1} \eta^{2} \Phi(2)|D u|^{p-2}\left|D^{2} u\right|^{2} d x .
\end{aligned}
$$

Inserting the estimate (3.13) in (3.12), we obtain that

$$
\begin{aligned}
\left|I_{6}\right| & \leq 2 \varepsilon \int_{\Omega} \eta^{2} \Phi\left((|D u|-1)_{+}\right)|D u|^{p-2}\left|D^{2} u\right|^{2} d x \\
& +\frac{\varepsilon \delta}{2} \int_{|D u| \geq 1} \eta^{2} \Phi(2)|D u|^{p-2}\left|D^{2} u\right|^{2} d x \\
& +c_{\gamma} C_{\varepsilon} \int_{\Omega} \eta^{2} \Phi^{\prime}\left((|D u|-1)_{+}\right) k^{2}(x)\left(\delta+(|D u|-1)_{+}\right)^{-1}|D u|^{p+2} d x .
\end{aligned}
$$

Since the equality (3.3) can be written as follows

$$
I_{2}+I_{3}=-I_{1}-I_{4}-I_{5}-I_{6},
$$

by virtue of (3.7), we get

$$
I_{2} \leq\left|I_{1}\right|+\left|I_{4}\right|+\left|I_{5}\right|+\left|I_{6}\right|
$$

and therefore, recalling the estimates (3.4), (3.8), (3.9) and (3.14), we get

$$
\begin{aligned}
& (1-\varepsilon) \int_{\Omega} \eta^{2} \Phi\left((|D u|-1)_{+}\right) \sum_{i, j, s, \alpha, \beta} f_{\xi_{i}^{\alpha} \xi_{j}^{\beta}}(x, D u) u_{x_{s} x_{i}}^{\alpha} u_{x_{s} x_{j}}^{\beta} d x \\
& \leq C_{\varepsilon} \int_{\Omega}|D \eta|^{2} \Phi\left((|D u|-1)_{+}\right)|D u|^{p} d x \\
& +2^{p-1} \int_{\Omega}(\eta|D \eta|) k(x) \Phi\left((|D u|-1)_{+}\right)|D u|^{p} d x \\
& +C_{\varepsilon} \int_{\Omega} \eta^{2} \Phi\left((|D u|-1)_{+}\right) k^{2}(x)|D u|^{p} d x
\end{aligned}
$$




$$
\begin{aligned}
& +3 \varepsilon \int_{\Omega} \eta^{2} \Phi\left((|D u|-1)_{+}\right)|D u|^{p-2}\left|D^{2} u\right|^{2} d x \\
& +\frac{\varepsilon \delta}{2} \int_{|D u| \geq 1} \eta^{2} \Phi(2)|D u|^{p-2}\left|D^{2} u\right|^{2} d x \\
& +c_{\gamma} C_{\varepsilon} \int_{\Omega} \eta^{2} \Phi^{\prime}\left((|D u|-1)_{+}\right) k^{2}(x)\left(\delta+(|D u|-1)_{+}\right)^{-1}|D u|^{p+2} d x .
\end{aligned}
$$

By the assumption (A3), the integral in the left hand side of (3.15) can be estimated as

$$
\int_{\Omega} \eta^{2} \Phi\left((|D u|-1)_{+}\right) \sum_{i, j, s, \alpha, \beta} f_{\xi_{i}^{\alpha} \xi_{j}^{\beta}}(x, D u) u_{x_{s} x_{i}}^{\alpha} u_{x_{s} x_{j}}^{\beta} d x \geq \nu \int_{\Omega} \eta^{2} \Phi\left((|D u|-1)_{+}\right)|D u|^{p-2}\left|D^{2} u\right|^{2} d x .
$$

Hence

$$
\begin{aligned}
& (1-\varepsilon) \nu \int_{\Omega} \eta^{2} \Phi\left((|D u|-1)_{+}\right)|D u|^{p-2}\left|D^{2} u\right|^{2} d x \\
\leq & 3 \varepsilon \int_{\Omega} \eta^{2} \Phi\left((|D u|-1)_{+}\right)|D u|^{p-2}\left|D^{2} u\right|^{2} d x \\
+ & C \int_{\Omega}|D \eta|^{2} \Phi\left((|D u|-1)_{+}\right)|D u|^{p} d x+C \int_{\Omega} \eta|D \eta| k(x) \Phi\left((|D u|-1)_{+}\right)|D u|^{p} d x \\
+ & C \int_{\Omega} \eta^{2} \Phi\left((|D u|-1)_{+}\right) k^{2}(x)|D u|^{p} d x+\frac{\varepsilon \delta}{2} \int_{|D u| \geq 1} \eta^{2} \Phi(2)|D u|^{p-2}\left|D^{2} u\right|^{2} d x \\
+ & C(1+\gamma) \int_{\Omega} \eta^{2} \Phi^{\prime}\left((|D u|-1)_{+}\right) k^{2}(x)\left(\delta+(|D u|-1)_{+}\right)^{-1}|D u|^{p+2} d x,
\end{aligned}
$$

with $C=C\left(n, N, p, \nu, L, L_{1}, \varepsilon\right)$. Choosing $\varepsilon=\frac{\nu}{6+\nu}$, we can reabsorb the first integral in the right hand side of the previous inequality by the left hand side, thus getting

$$
\begin{aligned}
& \int_{\Omega} \eta^{2} \Phi\left((|D u|-1)_{+}\right)|D u|^{p-2}\left|D^{2} u\right|^{2} d x \\
\leq & C \int_{\Omega}|D \eta|^{2} \Phi\left((|D u|-1)_{+}\right)|D u|^{p} d x+C \int_{\Omega} \eta|D \eta| k(x) \Phi\left((|D u|-1)_{+}\right)|D u|^{p} d x \\
+ & C \int_{\Omega} \eta^{2} \Phi\left((|D u|-1)_{+}\right) k^{2}(x)|D u|^{p} d x+C \delta \int_{|D u| \geq 1} \eta^{2} \Phi(2)|D u|^{p-2}\left|D^{2} u\right|^{2} d x \\
+ & C(1+\gamma) \int_{\Omega} \eta^{2} \Phi^{\prime}\left((|D u|-1)_{+}\right) k^{2}(x)\left(\delta+(|D u|-1)_{+}\right)^{-1}|D u|^{p+2} d x .
\end{aligned}
$$

Using (3.11), we get

$$
\begin{aligned}
& \Phi^{\prime}\left((|D u|-1)_{+}\right)\left(\delta+(|D u|-1)_{+}\right)^{-1} \\
& =\Phi^{\prime}\left((|D u|-1)_{+}\right)\left((|D u|-1)_{+}\right)^{-1} \frac{(|D u|-1)_{+}}{\delta+(|D u|-1)_{+}} \\
& \leq 2(\gamma+1) \Phi\left((|D u|-1)_{+}\right)\left((|D u|-1)_{+}\right)^{-2} \\
& \leq 2(\gamma+1)\left(1+(|D u|-1)_{+}\right)^{\gamma-2} \quad \text { a.e. in }\{|D u|>1\}
\end{aligned}
$$

where we used also that

$$
\frac{(|D u|-1)_{+}}{\delta+(|D u|-1)_{+}} \leq 1 \quad \forall \delta>0
$$


and the definition of $\Phi$. Hence, taking into account that $\Phi^{\prime}(0)=0$, we obtain that

$$
\begin{aligned}
& \int_{\Omega} \eta^{2} \Phi^{\prime}\left((|D u|-1)_{+}\right) k^{2}(x)\left(\delta+(|D u|-1)_{+}\right)^{-1}|D u|^{p+2} d x \\
\leq & C(1+\gamma) \int_{\Omega} \eta^{2} k^{2}(x)\left(1+(|D u|-1)_{+}\right)^{\gamma+p} d x .
\end{aligned}
$$

Inserting (3.17) in (3.16), we obtain

$$
\begin{aligned}
& \int_{\Omega} \eta^{2} \Phi\left((|D u|-1)_{+}\right)|D u|^{p-2}\left|D^{2} u\right|^{2} d x \\
\leq & C \int_{\Omega}|D \eta|^{2} \Phi\left((|D u|-1)_{+}\right)|D u|^{p} d x+C \int_{\Omega} \eta|D \eta| k(x) \Phi\left((|D u|-1)_{+}\right)|D u|^{p} d x \\
+ & C \int_{\Omega} \eta^{2} \Phi\left((|D u|-1)_{+}\right) k^{2}(x)|D u|^{p} d x+C \delta \int_{|D u| \geq 1} \eta^{2} \Phi(2)|D u|^{p-2}\left|D^{2} u\right|^{2} d x \\
+ & C(\gamma+1)^{2} \int_{\Omega} \eta^{2} k^{2}(x)\left(1+(|D u|-1)_{+}\right)^{\gamma+p} d x .
\end{aligned}
$$

Since

$$
\begin{aligned}
& \int_{\Omega} \eta|D \eta| k(x) \Phi\left((|D u|-1)_{+}\right)|D u|^{p} d x \\
& =\int_{\Omega}\left\{\eta^{2} k^{2}(x) \Phi\left((|D u|-1)_{+}\right)|D u|^{p}\right\}^{\frac{1}{2}}\left\{|D \eta|^{2} \Phi\left((|D u|-1)_{+}\right)|D u|^{p}\right\}^{\frac{1}{2}} d x \\
& \leq \frac{1}{2} \int_{\Omega} \eta^{2} k^{2}(x)\left(1+(|D u|-1)_{+}\right)^{\gamma+p} d x+\frac{1}{2} \int_{\Omega}|D \eta|^{2}\left(1+(|D u|-1)_{+}\right)^{\gamma+p} d x,
\end{aligned}
$$

and noting that, by Lemma 3.3 in [14], $|D u|^{p-2}\left|D^{2} u\right|^{2} \chi_{\{|D u| \geq 1\}} \in L_{\text {loc }}^{1}(\Omega)$, we can let $\delta$ go to 0 in (3.18), thus obtaining

$$
\begin{aligned}
& \int_{\Omega} \eta^{2} \Phi\left((|D u|-1)_{+}\right)|D u|^{p-2}\left|D^{2} u\right|^{2} d x \\
& \leq C \int_{\Omega}|D \eta|^{2}\left(1+(|D u|-1)_{+}\right)^{\gamma+p} d x+C(\gamma+1)^{2} \int_{\Omega} \eta^{2} k(x)^{2}\left(1+(|D u|-1)_{+}\right)^{\gamma+p} d x
\end{aligned}
$$

with $C=C\left(n, N, L, L_{1}, \nu\right)$. Estimate (3.1) follows recalling the definition of $\Phi$.

Step 2. In this step we prove that for all $x_{0} \in \Omega$ there exists $R_{\gamma}>0$ such that $B_{R_{\gamma}}\left(x_{0}\right) \Subset \Omega$ and for every $\rho$ and $R, 0<\rho<R<R_{\gamma}$,

$$
\int_{B_{\rho}\left(x_{0}\right)}\left|\left(1+(|D u|-1)_{+}\right)\right|^{\frac{(\gamma+p) n}{n-2}} d x \leq \frac{C(\gamma+p)^{\frac{2 n}{n-2}}}{(R-\rho)^{\frac{2 n}{n-2}}}\left(\int_{B_{R}\left(x_{0}\right)}\left(1+(|D u|-1)_{+}\right)^{\gamma+p} d x\right)^{\frac{n}{n-2}},
$$

for some $C=C\left(n, N, L, L_{1}, \nu\right)$.

Fix radii $0<\rho<s<t<R$, with $B_{R}\left(x_{0}\right) \Subset \Omega$, and a cut off function $\eta \in C_{0}^{\infty}\left(B_{t}\right)$ such that $\eta=1$ on $B_{s}$ and $|D \eta| \leq \frac{2}{t-s}$. With this choice of $\eta$, estimate (3.1) becomes

$$
\begin{aligned}
& \int_{B_{t}} \eta^{2}\left(1+(|D u|-1)_{+}\right)^{\gamma-2}\left((|D u|-1)_{+}\right)^{2}|D u|^{p-2}\left|D^{2} u\right|^{2} d x \\
\leq & C(\gamma+1)^{2} \int_{B_{t}} \eta^{2} k^{2}(x)\left(1+(|D u|-1)_{+}\right)^{\gamma+p} d x
\end{aligned}
$$




$$
\begin{aligned}
& +\frac{C}{(t-s)^{2}} \int_{B_{t} \backslash B_{s}}\left(1+(|D u|-1)_{+}\right)^{\gamma+p} d x \\
& \leq C(\gamma+1)^{2}\left(\int_{B_{t}} k^{n}(x) d x\right)^{\frac{2}{n}}\left(\int_{B_{t}}\left(1+(|D u|-1)_{+}\right)^{\frac{(\gamma+p) n}{n-2}} d x\right)^{\frac{n-2}{n}} \\
& +\frac{C}{(t-s)^{2}} \int_{B_{t} \backslash B_{s}}\left(1+(|D u|-1)_{+}\right)^{\gamma+p} d x,
\end{aligned}
$$

where we used the assumption $k \in L_{\text {loc }}^{n}(\Omega)$ and Hölder's inequality with exponents $\frac{n}{2}, \frac{n}{n-2}$. Following [14], we introduce the function

$$
G(t)=1+\int_{0}^{t}(1+s)^{\frac{\gamma+p-4}{2}} s d s
$$

and we note that

$$
\frac{1}{2(\gamma+p)}(1+t)^{\frac{\gamma+p}{2}} \leq G(t) \leq 2(1+t)^{\frac{\gamma+p}{2}}, \quad G^{\prime}(t)=t(1+t)^{\frac{\gamma+p-4}{2}} .
$$

Using the Sobolev imbedding Theorem for the function $\eta G\left((|D u|-1)_{+}\right)$, we get

$$
\begin{aligned}
& \left(\int_{\Omega}\left|\eta G\left((|D u|-1)_{+}\right)\right|^{\frac{2 n}{n-2}} d x\right)^{\frac{n-2}{n}} \leq c \int_{\Omega}\left|D\left(\eta G\left((|D u|-1)_{+}\right)\right)\right|^{2} d x \\
\leq & \left.c \int_{\Omega}|D \eta|^{2}\left(G\left((|D u|-1)_{+}\right)\right)^{2} d x+c \int_{\Omega} \eta^{2} \mid G^{\prime}\left((|D u|-1)_{+}\right)\right)\left.\right|^{2}\left|D\left((|D u|-1)_{+}\right)\right|^{2} d x .
\end{aligned}
$$

Using the properties of $G(t)$ at (3.21) in the previous inequality, we obtain

$$
\begin{aligned}
& \frac{1}{(\gamma+p)^{2}}\left(\int_{\Omega} \eta^{\frac{2 n}{n-2}}\left(1+(|D u|-1)_{+}\right)^{\frac{(\gamma+p) n}{n-2}} d x\right)^{\frac{n-2}{n}} \\
\leq & c \int_{\Omega}|D \eta|^{2}\left(1+(|D u|-1)_{+}\right)^{\gamma+p} d x \\
+ & c \int_{\Omega} \eta^{2}\left(1+(|D u|-1)_{+}\right)^{\gamma+p-4}\left((|D u|-1)_{+}\right)^{2}\left|D\left((|D u|-1)_{+}\right)\right|^{2} d x \\
\leq & \frac{c}{(t-s)^{2}} \int_{B_{R}}\left(1+(|D u|-1)_{+}\right)^{\gamma+p} d x \\
+ & c \int_{B_{t}} \eta^{2}\left(1+(|D u|-1)_{+}\right)^{\gamma-2}\left|(|D u|-1)_{+}\right|^{2}|D u|^{p-2}\left|D^{2} u\right|^{2} d x .
\end{aligned}
$$

Combining estimates (3.20) and (3.22), we obtain

$$
\begin{aligned}
& \int_{B_{s}}\left(1+(|D u|-1)_{+}\right)^{\frac{(\gamma+p) n}{n-2}} d x \\
\leq & c(\gamma+p)^{\frac{4 n}{n-2}}\left(\int_{B_{R}} k(x)^{n} d x\right)^{\frac{2}{n-2}} \int_{B_{t}}\left(1+(|D u|-1)_{+}\right)^{\frac{(\gamma+p) n}{n-2}} d x \\
+ & \frac{c(\gamma+p)^{\frac{2 n}{n-2}}}{(t-s)^{\frac{2 n}{n-2}}}\left(\int_{B_{R}}\left(1+(|D u|-1)_{+}\right)^{\gamma+p} d x\right)^{\frac{n}{n-2}} .
\end{aligned}
$$


By the absolute continuity of the integral we can choose a radius $R_{\gamma}$, which obviously depends also on $n, N, p, \nu, L, L_{1}$, such that

$$
c(\gamma+p)^{\frac{4 n}{n-2}}\left(\int_{B_{R_{\gamma}}} k(x)^{n} \mathrm{~d} x\right)^{\frac{2}{n-2}} \leq \frac{1}{2}
$$

so that, if $R<R_{\gamma}$, estimate (3.23) becomes

$$
\begin{aligned}
\int_{B_{s}}\left(1+(|D u|-1)_{+}\right)^{\frac{(\gamma+p) n}{n-2}} d x & \leq \frac{1}{2} \int_{B_{t}}\left(1+(|D u|-1)_{+}\right)^{\frac{(\gamma+p) n}{n-2}} d x \\
& +\frac{c(\gamma+p)^{\frac{2 n}{n-2}}}{(t-s)^{\frac{2 n}{n-2}}}\left(\int_{B_{R}}\left(1+(|D u|-1)_{+}\right)^{\gamma+p} d x\right)^{\frac{n}{n-2}} .
\end{aligned}
$$

Since the previous inequality is valid for every radii $\rho<s<t<R<R_{\gamma}$, we can use the iteration Lemma 2.1, so obtaining (3.19).

Step 3. In this step we conclude the proof.

Fix $q>p$. Let $i_{*}$ be the unique integer such that $\log _{\frac{n}{n-2}} \frac{q}{p}-1 \leq i_{*}<\log _{\frac{n}{n-2}} \frac{q}{p}$. If we define the increasing sequence of exponents $p_{j}, j \in\left\{0,1, \cdots, i_{*}+1\right\}$ by setting

$$
p_{j}:=\left(\frac{n}{n-2}\right)^{j} p
$$

we obtain $p_{i_{*}}<q \leq p_{i_{*}+1}$.

For every $j \in\left\{0,1, \cdots, i_{*}\right\}$ define $\gamma_{j}:=p_{j}-p$. By Step 2 there exist $R_{\gamma_{j}}>0$ and $C>0$ such that for all $0<\rho<R<R_{\gamma_{j}}$ (3.19) holds with $\gamma$ replaced by $\gamma_{j}$. Note that since $R_{\gamma_{j}}$ satisfies (3.24), then we also have that

$$
c p^{\frac{4 n}{n-2}}\left(\int_{B_{r}} k(x)^{n} \mathrm{~d} x\right)^{\frac{2}{n-2}} \leq \frac{1}{2}
$$

for every $0<r \leq R_{\gamma_{j}}$.

Define $R_{*}:=\min \left\{R_{j}: j=1, \cdots, i_{*}\right\}>0$. Fix $0<\rho<R<R_{*}$ and let us define the decreasing sequence of radii $\rho_{j}$

$$
\rho_{j}:=\rho+\frac{R-\rho}{2^{j}}, \quad j \in\left\{0,1, \cdots, i_{*}\right\},
$$

so that $\rho_{0}=R>\rho_{j}>\rho_{j+1}>\rho$. By (3.25), with $\sigma=p_{j}$, and (3.19) we get

$$
\left(\int_{B_{\rho_{j+1}}}\left(1+(|D u|-1)_{+}\right)^{p_{j+1}} d x\right)^{\frac{1}{p_{j+1}}} \leq \frac{C_{p_{j}}}{\left(\rho_{j}-\rho_{j+1}\right)^{\frac{2}{p_{j}}}}\left(\int_{B_{\rho_{j}}}\left(1+(|D u|-1)_{+}\right)^{p_{j}} d x\right)^{\frac{1}{p_{j}}}
$$

where we have chosen $\rho=\rho_{j+1}, R=\rho_{j}$ and $\gamma+p=p_{j}$. Iterating (3.26), we deduce

$$
\left(\int_{B_{\rho}}\left(\left(1+(|D u|-1)_{+}\right)\right)^{p_{i_{*}+1}} d x\right)^{\frac{1}{p_{i_{*}+1}}} \leq \tilde{C}\left(\int_{B_{R}}\left(1+(|D u|-1)_{+}\right)^{p} d x\right)^{\frac{1}{p}}
$$

where

$$
\tilde{C}=\prod_{j=0}^{i_{*}} \frac{C_{p_{j}}}{\left(\rho_{j}-\rho_{j+1}\right)^{\frac{2}{p_{j}}}}=: C\left(n, N, p, q, L, L_{1}, \nu, \rho, R\right) .
$$

Observing that $q \leq p_{i_{*}+1}$ and by using the left inequality in (A2), the conclusion follows. 


\section{Proof of Theorem 1.1}

Before going on with the proof of the main Theorem, we state an approximation result for the energy density $f$ through a sequence of uniformly elliptic integrands having Lipschitz dependence on the $x$ variable.

We recall that, without loss of generality, we assumed that the radius $\tilde{R}$ appearing in the assumptions (A1)-(A5) is equal to 1.

Proposition 4.1. Let $f: \Omega \times \mathbb{R}^{n N} \rightarrow[0,+\infty)$ be a Carathéodory function convex and $C^{2}$ with respect to the last variable and satisfying assumptions (A1)-(A5). Fixed an open set $\Omega^{\prime} \Subset \Omega$, there exists a sequence of $C^{2}$-functions $f_{h}: \Omega^{\prime} \times \mathbb{R}^{n N} \rightarrow[0,+\infty), f_{h}$ convex in the last variable, such that $f_{h}$ converges to $f$ pointwise a.e. on $\Omega^{\prime}$ and everywhere in $\mathbb{R}^{N}$. Moreover, $f_{h}$ satisfies the following properties:

$(\mathcal{A} 1)$ for every $x \in \Omega^{\prime}$ and $\xi \in \mathbb{R}^{n N} \backslash B_{2}(0), f_{h}(x, \xi)=\tilde{f}_{h}(x,|\xi|)$,

$(\mathcal{A} 2)$ there exist $\tilde{L}, \tilde{c}_{1}, \tilde{c}_{2}>0$, independent of $h$, such that for all $(x, \xi) \in \Omega^{\prime} \times \mathbb{R}^{n N}$

$$
\tilde{c}_{1}|\xi|^{p}-\tilde{c}_{2} \leq f_{h}(x, \xi) \leq \tilde{L}(1+|\xi|)^{p},
$$

(A3) there exists $\tilde{\nu}$, depending on $\nu$ and $p$, but not on $h$, such that for every $x \in \Omega^{\prime}, \xi \in \mathbb{R}^{n N} \backslash B_{2}(0)$ and $\lambda \in \mathbb{R}^{n N}$

$$
\tilde{\nu}(1+|\xi|)^{p-2}|\lambda|^{2} \leq\left\langle D_{\xi \xi} f_{h}(x, \xi) \lambda, \lambda\right\rangle,
$$

(A4) there exists $\tilde{L}_{1}>0$, independent of $h$, such that for all $(x, \xi) \in \Omega^{\prime} \times \mathbb{R}^{n N} \backslash B_{2}(0)$

$$
\left|D_{\xi \xi} f_{h}(x, \xi)\right| \leq \tilde{L}_{1}(1+|\xi|)^{p-2},
$$

(A5) for every $x \in \Omega^{\prime}$ and $\xi \in \mathbb{R}^{n N} \backslash B_{2}(0)$,

$$
\left|D_{\xi x} f_{h}(x, \xi)\right| \leq 2^{p-1} k_{h}(x)(1+|\xi|)^{p-1},
$$

where $k_{h} \in C^{\infty}\left(\Omega^{\prime}\right)$ is a non-negative function, such that $k_{h} \rightarrow k$ in $L^{n}\left(\Omega^{\prime}\right)$,

(H1) there exists $\Lambda_{h}>0$ such that for every $x \in \Omega^{\prime}$ and $\xi \in \overline{B_{2}(0)}$

$$
\left|D_{\xi x} f_{h}(x, \xi)\right| \leq \Lambda_{h}(1+|\xi|)^{p-1} .
$$

(H2) there exists $\mu_{h}>0$ such that for all $(x, \xi) \in \Omega^{\prime} \times \mathbb{R}^{n N}$ and for all $\lambda \in \mathbb{R}^{n N}$

$$
\mu_{h}(1+|\xi|)^{p-2}|\lambda|^{2} \leq\left\langle D_{\xi \xi} f_{h}(x, \xi) \lambda, \lambda\right\rangle .
$$

$(\mathcal{H} 3)$ there exists $\sigma_{h}>0$ such that for all $(x, \xi) \in \Omega^{\prime} \times \mathbb{R}^{n N}$

$$
\left|D_{\xi \xi} f_{h}(x, \xi)\right| \leq \sigma_{h}(1+|\xi|)^{p-2} .
$$

Proof. To prove $(\mathcal{A} 1),(\mathcal{A} 2)$ and $(\mathcal{H} 2)$ we argue as in the proof of Lemma 4.2 in [9], but replacing the function $f_{k}$ and the exponent $q$ with $f$ and $p$, respectively. For the sake of completeness, we report the complete proof.

Denote $B_{1}^{n} \subset \mathbb{R}^{n}$ and $B_{1}^{n N} \subset \mathbb{R}^{n N}$ the unit balls centered in 0 . Fixed a positive decreasing sequence $\varepsilon_{h} \rightarrow 0$, with $h \geq h_{0}$, where $h_{0}$ is large enough to have $x+\varepsilon_{h} y \in \Omega$ for every $x \in \Omega^{\prime}$ and every $y \in B_{1}^{n}$. Without loss of generality we assume $\varepsilon_{h} \leq \frac{1}{2}$.

Let $\sigma: B_{1}^{n} \rightarrow[0,+\infty)$ and $\rho: B_{1}^{n N} \rightarrow[0,+\infty)$ be two radially symmetric mollifiers such that $\int_{B_{1}^{n}} \sigma=1$ and $\int_{B_{1}^{n N}} \rho=1$. Define $F_{h}: \Omega^{\prime} \times \mathbb{R}^{n N} \rightarrow[0,+\infty)$,

$$
F_{h}(x, \xi):=\int_{B_{1}^{n}} \int_{B_{1}^{n N}} \sigma(y) \rho(\eta) f\left(x+\varepsilon_{h} y, \xi+\varepsilon_{h} \eta\right) d \eta d y
$$

and

$$
f_{h}(x, \xi):=F_{h}(x, \xi)+\varepsilon_{h}(1+|\xi|)^{p}
$$


For each $h, f_{h} \in C^{2}$, one can easily check that $f_{h}$ is convex in the last variable and, up to subsequences, $f_{h}$ converges pointwise a.e. to $f$ in $\Omega^{\prime}$ and everywhere in $\mathbb{R}^{N}$. Moreover, by (A1), $f_{h}(x, \cdot)$ is radial with respect to $\xi$ in $\mathbb{R}^{n N} \backslash B_{2}^{n N}$.

The property $(\mathcal{A} 2)$ is a trivial consequence of the assumption (A2).

To prove $(\mathcal{A} 3)$ it is enough to prove that a similar inequality holds for $F_{h}$. By (A3)

$$
\left\langle D_{\xi \xi} F_{h}(x, \xi) \lambda, \lambda\right\rangle \geq \nu \int_{B_{1}^{n}} \int_{B_{1}^{n N}} \sigma(y) \rho(\eta)\left(1+\left|\xi+\varepsilon_{h} \eta\right|\right)^{p-2} d \eta d y|\lambda|^{2} .
$$

Since

$$
\left(1+\left|\xi+\varepsilon_{h} \eta\right|\right)^{p-2} \geq c_{p}(1+|\xi|)^{p-2}
$$

where $c_{p}=\min \left\{\frac{1}{2^{p-2}},\left(\frac{3}{2}\right)^{p-2}\right\}$, we can conclude using the properties of $\sigma$ and $\rho$.

To prove property $(\mathcal{A} 4)$ we observe that for all $\xi$, with $|\xi| \geq 2$ we have

$$
\left|D_{\xi \xi} f_{h}(x, \xi)\right| \leq \int_{B_{1}^{n}} \int_{B_{1}^{n N}} \sigma(y) \rho(\eta)\left|D_{\xi \xi} f\left(x+\varepsilon_{h} y, \xi+\varepsilon_{h} \eta\right)\right| d \eta d y+\frac{1}{2}\left|D_{\xi \xi}\left((1+|\xi|)^{p}\right)\right|
$$

and we conclude using (A4), the inequality

$$
\left(1+\left|\xi+\varepsilon_{h} \eta\right|\right)^{p-2} \leq c_{p}^{\prime}(1+|\xi|)^{p-2}
$$

and the properties of $\sigma$ and $\rho$.

Let us prove ( $(\mathcal{A 5})$. By the assumption (A5), for all $|\xi|>2$, we have

$$
\begin{aligned}
& \left|D_{\xi x} f_{h}(x, \xi)\right|=\left|D_{\xi x} F_{h}(x, \xi)\right| \leq \int_{B_{1}^{n}} \int_{B_{1}^{n N}} \sigma(y) \rho(\eta)\left|D_{\xi x} f\left(x+\varepsilon_{h} y, \xi+\varepsilon_{h} \eta\right)\right| d \eta d y \\
& \leq \int_{B_{1}^{n}} \int_{B_{1}^{n N}} \sigma(y) \rho(\eta) k\left(x+\varepsilon_{h} y\right)\left(1+\left|\xi+\varepsilon_{h} \eta\right|\right)^{p-1} d \eta d y \leq 2^{p-1} k_{h}(x)(1+|\xi|)^{p-1},
\end{aligned}
$$

where

$$
k_{h}(x):=\int_{B_{1}^{n}} \sigma(y) k\left(x+\varepsilon_{h} y\right) d y
$$

is a smooth function, satisfying $k_{h} \rightarrow k$ in $L^{n}\left(\Omega^{\prime}\right)$. Thus, (A5) follows.

To prove (H1), we observe that, for all $x \in \Omega^{\prime}$ and $\xi \in B_{2}^{n N}$, thanks to assumption (A2), it holds the following

$$
\begin{aligned}
& \left|D_{\xi x} f_{h}(x, \xi)\right|=\left|D_{\xi x} F_{h}(x, \xi)\right| \\
& =\varepsilon_{h}^{-n} \varepsilon_{h}^{-n N}\left|D_{\xi x} \int_{B_{\varepsilon_{h}}^{n}(x)} \int_{B_{\varepsilon_{h}}^{n N}(\xi)} \sigma\left(\frac{w-x}{\varepsilon_{h}}\right) \rho\left(\frac{z-\xi}{\varepsilon_{h}}\right) f(w, z) d w d z\right| \\
& \leq C(L, \Omega) \varepsilon_{h}^{-n-n N-2}\left\|D_{\xi} \rho\right\|_{L^{\infty}\left(B_{1}^{n N}\right)}\left\|D_{x} \sigma\right\|_{L^{\infty}\left(B_{1}^{n}\right)} \int_{B_{\varepsilon_{h}}^{n N}(\xi)}(1+|z|)(1+|z|)^{p-1} d z \\
& \leq C(L, \Omega)(1+|\xi|)^{p-1} \varepsilon_{h}^{-n-n N-2}\left\|D_{\xi} \rho\right\|_{L^{\infty}\left(B_{1}^{n N}\right)}\left\|D_{x} \sigma\right\|_{L^{\infty}\left(B_{1}^{n}\right)} \int_{B_{3}^{n N}(0)}(1+|z|) d z .
\end{aligned}
$$

Therefore, we get

$$
\left|D_{\xi x} f_{h}(x, \xi)\right| \leq C(L, \Omega) \varepsilon_{h}^{-n-n N-2} 4^{n N+1}\|D \sigma\|_{\infty}\|D \rho\|_{\infty}(1+|\xi|)^{p-1},
$$

and $(\mathcal{H} 1)$ follows.

As far as the property $(\mathcal{H} 2)$ is concerned, notice that $F_{h}$ are convex functions, because $f$ is convex, therefore

$$
\left\langle D_{\xi \xi} f_{h}(x, \xi) \lambda, \lambda\right\rangle \geq \varepsilon_{h}\left\langle D_{\xi \xi}\left(\left(1+|\xi|^{2}\right)^{\frac{p}{2}}\right) \lambda, \lambda\right\rangle
$$


and we immediately conclude.

Since $(\mathcal{A} 4)$ holds, the property $(\mathcal{H} 3)$ follows observing that the second order derivatives with respect to $\xi$ of $f_{h}(x, \xi)$ are equi-continuous in $\Omega^{\prime} \times \overline{B_{2}(0)}$.

We are now ready to prove our main result.

Proof of Theorem 1.1. Let $u$ be a local minimizer of the functional $\mathcal{F}$ in (1.1). Fixed $\Omega^{\prime} \Subset \Omega$, let $f_{h}$ be the sequence of functions given by Proposition 4.1.

Fix a ball $B_{r}\left(x_{0}\right)$ in $\Omega^{\prime}$, such that $r<R_{\gamma}$ where $R_{\gamma}$ is the radius determined in Theorem 3.1. By virtue of $(\mathcal{A} 2)$, we have the existence of a weak solution $v_{h} \in W^{1, p}\left(B_{r}\left(x_{0}\right), \mathbb{R}^{N}\right)$ of the problem

$$
\min \left\{\mathcal{F}_{h}\left(w ; B_{r}\left(x_{0}\right)\right):=\int_{B_{r}\left(x_{0}\right)} f_{h}(x, D w) d x: w \in u+W_{0}^{1, p}\left(B_{r}\left(x_{0}\right), \mathbb{R}^{N}\right)\right\} .
$$

From the first inequality in $(\mathcal{A} 2)$ and the minimality of $v_{h}$, there exists $c>0$, independent of $h$, such that

$$
\int_{B_{r}\left(x_{0}\right)}\left|D v_{h}\right|^{p} d x \leq c \int_{B_{r}\left(x_{0}\right)}\left(1+f_{h}\left(x, D v_{h}\right)\right) d x \leq c \int_{B_{r}\left(x_{0}\right)}\left(1+f_{h}(x, D u)\right) d x .
$$

By the right inequality in $(\mathcal{A} 2)$ and the dominated convergence theorem, we have

$$
\limsup _{h \rightarrow+\infty} \int_{B_{r}\left(x_{0}\right)}\left|D v_{h}\right|^{p} d x \leq c \lim _{h} \int_{B_{r}\left(x_{0}\right)}\left(1+f_{h}(x, D u)\right) d x \leq c \int_{B_{r}\left(x_{0}\right)}(1+f(x, D u)) d x .
$$

Therefore, up to a subsequence, $v_{h}$ weakly converges in $W^{1, p}$ to a function $v \in u+W_{0}^{1, p}\left(B_{r}\left(x_{0}\right)\right)$. We now claim that $v$ is a minimizer of $\mathcal{F}\left(\cdot ; B_{r}\left(x_{0}\right)\right)$. By the convexity of the functions $f_{h}(x, \cdot)$ and $(\mathcal{A} 2)$, the sequence $\left\{\mathcal{F}_{h}\left(\cdot ; B_{r}\left(x_{0}\right)\right)\right\}_{h} \Gamma$-converges to $\mathcal{F}\left(\cdot ; B_{r}\left(x_{0}\right)\right)$ with respect to the weak $W^{1, p}$-topology induced on $u+W_{0}^{1, p}\left(B_{r}\left(x_{0}\right)\right)$ (see Theorem 5.14 in [12]). Therefore, Corollary 7.20 in [12] implies that $v$ is a minimizer of $\mathcal{F}\left(\cdot ; B_{r}\left(x_{0}\right)\right)$.

Fix $\rho<r$. We claim that $v \in W^{1, q}\left(B_{\rho}\left(x_{0}\right), \mathbb{R}^{N}\right)$ for all $q>p$ and that there exists $C>0$ such that

$$
\int_{B_{\rho}\left(x_{0}\right)}|D v|^{q} d x \leq C\left[\int_{B_{r}\left(x_{0}\right)}(1+f(x, D u)) d x\right]^{\frac{q}{p}} .
$$

Indeed, for all $h, f_{h}$ satisfies the assumptions of Lemma 2.3, with $\Omega$ replaced by $B_{r}\left(x_{0}\right)$, therefore $v_{h} \in W_{\text {loc }}^{1, \infty}\left(B_{r}\left(x_{0}\right)\right) \cap W_{\text {loc }}^{2,2}\left(B_{r}\left(x_{0}\right)\right)$ and $\left(1+\left|D v_{h}\right|^{2}\right)^{\frac{p-2}{2}}\left|D^{2} v_{h}\right|^{2} \in L_{\text {loc }}^{1}\left(B_{r}\left(x_{0}\right)\right)$. We are legitimate to apply the apriori estimate of Theorem 3.1 to $f_{h}$ and $v_{h}$, thus obtaining that $v_{h} \in W^{1, q}\left(B_{\rho}\left(x_{0}\right)\right)$ for all $q>p$ and that the following estimate

$$
\int_{B_{\rho}\left(x_{0}\right)}\left|D v_{h}\right|^{q} d x \leq C\left[\int_{B_{r}\left(x_{0}\right)}\left(1+f_{h}\left(x, D v_{h}\right)\right) d x\right]^{\frac{q}{p}}
$$

holds for a positive constant $C$. Note that, thanks to the definition of $f_{h}$ in (4.1) and since the constants $\tilde{L}, \tilde{\nu}, \tilde{L}_{1}$ in $(\mathcal{A} 2)-(\mathcal{A} 5)$ are independent of $h$, and since $k_{h}$ converges to $k$ in $L^{n}\left(\Omega^{\prime}\right)$, then the constant $C$ is independent of $h$.

It follows that, up to subsequences, $v_{h}$ weakly converges to $v$ in $W^{1, q}\left(B_{\rho}\left(x_{0}\right), \mathbb{R}^{N}\right)$ for every fixed $q>p$ and passing to the limit as $h \rightarrow+\infty$ in the previous estimate we obtain

$$
\int_{B_{\rho}\left(x_{0}\right)}|D v|^{q}, d x \leq \liminf _{h} \int_{B_{r}\left(x_{0}\right)}\left|D v_{h}\right|^{q} d x \leq C\left[\int_{B_{r}\left(x_{0}\right)}(1+f(x, D u)) d x\right]^{\frac{q}{p}} .
$$

Therefore (4.2) holds true.

We now compare the $L^{q}$-norm of $D u$ and $D v$. 
From Lemma 2.2 there exists $R_{0}=R_{0}(p, \nu, L)$ such that (2.1) holds. Thus,

$$
\begin{aligned}
\int_{B_{\rho}\left(x_{0}\right)}|D u|^{q} d x \leq \int_{B_{\rho}\left(x_{0}\right) \cap\left\{x:|D u+D v| \leq 2 R_{0}\right\}}|D u|^{q} d x+\int_{B_{\rho}\left(x_{0}\right) \cap\{x: D u=D v\}}|D u|^{q} d x \\
\quad \leq 2^{q-1} \int_{B_{\rho}\left(x_{0}\right) \cap\left\{x:|D u+D v| \leq 2 R_{0}\right\}}\left(|D u+D v|^{q}+|D v|^{q}\right) d x+\int_{B_{\rho}\left(x_{0}\right)}|D v|^{q} d x,
\end{aligned}
$$

which implies that there exists $C>0$, depending on $n, p, q, \nu, L, L_{1}$, such that

$$
\int_{B_{\rho}\left(x_{0}\right)}|D u|^{q} d x \leq C \int_{B_{\rho}\left(x_{0}\right)}\left(1+|D v|^{q}\right) d x .
$$

This inequality, together with (4.3) implies the thesis. The $\alpha$-Hölder continuity of $u$, for any exponent $\alpha<1$, comes from the classical Sobolev-Morrey embedding theorem.

\section{REFERENCES}

[1] E. Acerbi, N. Fusco: Regularity of minimizers of non-quadratic functionals: the case $1<p<2$, J. Math. Anal. Appl. 140 (1989) 115-135.

[2] E. Acerbi, N. Fusco: Partial regularity under anisotropic $(p, q)$ growth conditions, J. Differential Equations 107 (1994), no. $1,46-67$.

[3] L. Boccardo, P. Marcellini, C. Sbordone $L^{\infty}$-regularity for variational problems with sharp nonstandard growth conditions, Boll. Un. Mat. Ital. A 4 (1990) 219-225.

[4] P. Celada, G. Cupini, M. Guidorzi: Existence and regularity of minimizers of nonconvex integrals with $p-q$ growth, ESAIM Control Optim. Calc. Var. 13 (2007), 343-358.

[5] M. Chipot, L.C. Evans: Linearisation at infinity and Lipschitz estimates for certain problems in the calculus of variations. Proc. Roy. Soc. Edinburgh Sect. A 102 (1986), no. 3-4, 291-303.

[6] M. Colombo, G. Mingione: Regularity for double phase variational problems, Arch. Rat. Mech. Anal. 215 (2015) $443-496$.

[7] M. Colombo, G. Mingione: Bounded minimisers of double phase variational integrals, Arch. Rat. Mech. Anal. 218 (2015) 219-273.

[8] G. Cupini, N. Fusco, R. Petti: Hölder continuity of local minimizers, J. Math. Anal. Appl. 235 (1999) 578-597.

[9] G. Cupini, M. Guidorzi, E. Mascolo: Regularity of minimizers of vectorial integrals with $p-q$ growth Nonlinear Anal. 54 (2003) 591-616.

[10] G. Cupini, A.P. Migliorini: Hölder continuity for local minimizers of a nonconvex variational problem, J. Convex Anal. 10 (2003) 389-408.

[11] B. Dacorogna: Direct methods in the Calculus of Variations. 2nd edition. Appl. Math. Sci. 78, Springer, New York, 2008.

[12] G. Dal Maso: An introduction to Г-convergence, Progr. Nonlinear Differential Equations Appl., no. 8, Birkhäuser, Boston, 1993.

[13] E. De Giorgi: Un esempio di estremali discontinue per un problema variazionale di tipo ellittico, Boll. Unione Mat. Ital. IV. Ser. 1 (1968) 135-137.

[14] M. Eleuteri, P. Marcellini, E. Mascolo: Lipschitz estimates for systems with ellipticity conditions at infinity, Ann. Mat. Pura e Appl., to appear.

[15] L. Esposito, F. Leonetti, G. Mingione: Regularity results for minimizers of irregular integrals with $(p, q)$ growth, Forum Mathematicum 14 (2002) 245-272.

[16] L. Esposito, F. Leonetti, G. Mingione: Sharp regularity for functionals with (p,q) growth, J. Differential Equations 204 (2004) 5-55.

[17] L. Esposito, G. Mingione, C. Trombetti: On the Lipschitz regularity for certain elliptic problems Forum Math. 18 (2006) 263-292.

[18] I. Fonseca, N. Fusco, P. Marcellini: An existence result for a nonconvex variational problem via regularity, ESAIM: Control, Optim. Calc. Var. 7 (2002) 69-96.

[19] I. Fonseca, I. Malý, G. Mingione: Scalar minimizers with fractal singular sets, Arch. Rat. Mech. Anal. 172 (2004) 295307.

[20] K. Fey, M. Foss: Morrey regularity results for asymptotically convex variational problems with $(p, q)$ growth. J. Differential Equations 246 (2009) 4519-4551.

[21] M. Foss, C.S. Goodrich: Partial Hölder continuity of minimizers of functionals satisfying a general asymptotic relatedness condition J. Convex Anal. 22 (2015), 219-246. 
[22] M. Foss, A. Passarelli di Napoli, A. Verde: Morrey regularity and continuity results for almost minimizers of asymptotically convex integrals. Appl. Math. (Warsaw) 35 (2008), 335-353.

[23] M. Foss, A. Passarelli di Napoli, A. Verde: Global Morrey regularity results for asymptotically convex variational problems. Forum Math. 20 (2008), 921-953.

[24] M. Foss, A. Passarelli di Napoli, A. Verde: Global Lipschitz regularity for almost minimizers of asymptotically convex variational problems. Ann. Mat. Pura Appl. (4) 189 (2010), 127-162.

[25] N. Fusco, C. Sbordone: Local boundedness of minimizers in a limit case. Manuscripta Mathematica 69, (1990) 19-25.

[26] N. Fusco, C. Sbordone: Some remarks on the regularity of minima of anisotropic integrals. Commun. in Partial Diff. Equ. 18 (1993) 153-167.

[27] F. Giannetti: A $C^{1, \alpha}$ partial regularity result for integral functionals with $p(x)$ - growth condition. Adv. Calc. Var., to appear.

[28] F. Giannetti, A. Passarelli di Napoli: Hölder continuity of degenerate $p$-harmonic functions. Ann. Acad. Sci. Fennicae Math. 39 (2014) 567-577.

[29] F. Giannetti, A. Passarelli di Napoli: Higher differentiability of minimizers of variational integrals with variable exponents. Math. Zeitschrift (2015) 10.1007/s00209-015-1453-4.

[30] F. Giannetti, A. Passarelli di Napoli, C. Scheven: Higher differentiability of solutions of parabolic systems with discontinuous coefficients, Preprint (2015).

[31] M. Giaquinta, G. Modica: Remarks on the regularity of the minimizers of certain degenerate functionals, Manuscripta Math. 57 (1986) 55-99.

[32] R. Giova: Higher differentiability for n-harmonic systems with Sobolev coefficients J. Diff. Equations 259 (2015) 56675687.

[33] E. Giusti: Direct methods in the calculus of variations, World Scientific Publishing Co., Inc., River Edge, NJ, 2003.

[34] J. Kristensen, G. Mingione: Boundary regularity in variational problems, Arch. Ration. Mech. Anal. 198 (2010) 369-455.

[35] T. Kuusi, G. Mingione: Universal potential estimates, J. Funct. Anal. 262 (2012) 4205-4269.

[36] P. Marcellini: Regularity of minimizers of integrals of the calculus of variations with non standard growth conditions, Arch. Rat. Mech. Anal. 105 (1989) 267-284.

[37] P. Marcellini: Regularity and existence of solutions of elliptic equations with $p, q$-growth conditions, J. Differential Equations 90 (1991) 1-30.

[38] G. Mingione: Regularity of minima: an invitation to the dark side of the calculus of variations. Appl. Math. 51 (2006) $355-426$.

[39] C. Mooney, O. Savin: Some singular minimizers in low dimensions in the calculus of variations, arXiv:1503.00671 (2015).

[40] J. Nečas: Example of an irregular solution to a nonlinear elliptic system with analytic coefficients and conditions for regularity, Theory Nonlin. Oper., (Proc. Fourth Internat. Summer School, Acad. Sci., Berlin, 1975), 197-206. Abh. Akad. Wiss. DDR Abt. Math.-Natur.-Tech., Jahrgang 1977, 1, Akademie-Verlag, Berlin, 1977.

[41] A. Passarelli di Napoli: Higher differentiability of minimizers of variational integrals with Sobolev coefficients. Adv. Cal. Var. 7 (1) (2014), 59-89.

[42] A. Passarelli di Napoli: Higher differentiability of solutions of elliptic systems with Sobolev coefficients: the case $p=$ $n=2$. Pot. Anal. 41 (3) (2014), 715-735

[43] A. Passarelli di Napoli: Regularity results for non-autonomous variational integrals with discontinuous coefficients. Atti Accad. Naz. Lincei Rend. Lincei Mat. Appl. 26 (4) (2015), 475-496.

[44] A. Passarelli di Napoli, A. Verde: A regularity result for asymptotically convex problems with lower order terms. $J$. Convex Anal. 15 (2008), 131-148.

[45] V. Sveràk, X. Yan: Non-Lipschitz minimizers of smooth uniformly convex functionals Proc. Natl. Acad. Sci. USA 99 (24) (2002) 15269-15276.

Giovanni Cupini

Dipartimento di Matematica

Università di Bologna

Piazza di Porta S. Donato 5, 40126 Bologna, Italy.

giovanni.cupini@unibo.it

Flavia Giannetti

Dipartimento di Matematica e Appl. "R. Caccioppoli"

Università di Napoli "Federico II" 
Via Cintia, 80126 Napoli, Italy.

giannett@unina.it

\section{Raffaella Giova}

Dipartimento di Studi Economici e Giuridici

Università degli Studi di Napoli "Parthenope"

Palazzo Pacanowsky- Via Generale Parisi, 13, 80123 Napoli, Italy.

raffaella.giova@uniparthenope.it

Antonia Passarelli di Napoli

Dipartimento di Matematica e Appl. "R. Caccioppoli"

Università di Napoli "Federico II"

Via Cintia, 80126 Napoli, Italy.

antpassa@unina.it 